\title{
Effect of PET/CT on Management of Patients with Non-Small Cell Lung Cancer: Results of a Prospective Study with 5-Year Survival Data
}

\author{
Deborah L. Gregory ${ }^{1}$, Rodney J. Hicks², Annette Hogg ${ }^{2}$, David S. Binns ${ }^{2}$, Poh Lin Shum ${ }^{1}$, Alvin Milner ${ }^{3}$, Emma Link ${ }^{3}$, \\ David L Ball ${ }^{1}$, and Michael P. Mac Manus ${ }^{1}$ \\ ${ }^{I}$ Department of Radiation Oncology, Peter MacCallum Cancer Centre, Melbourne, Victoria, Australia; ${ }^{2}$ Centre for Cancer Imaging, \\ Peter MacCallum Cancer Centre, Melbourne, Victoria, Australia; and ${ }^{3}$ Centre for Biostatistics and Clinical Trials, Peter MacCallum \\ Cancer Centre, Melbourne, Victoria, Australia
}

We investigated the incremental management impact and prognostic value of staging with ${ }^{18} \mathrm{~F}-\mathrm{FDG} \mathrm{PET} / \mathrm{CT}$ in patients with non-small cell lung cancer (NSCLC) being considered for potentially curative therapies. Methods: Information on 168 consecutive patients with NSCLC being considered for surgery or definitive radiotherapy with curative intent before PET/CT was entered into a prospective database. The pre-PET/CT management plan, based on conventional imaging (conventional CT, appropriately supplemented by bone scintigraphy or other modalities), was defined prospectively by referring clinicians before PET/CT results became available. After PET/ CT, actual clinical management was recorded, and patients were followed up until 5 y or death. The appropriateness of $\mathrm{PET} / \mathrm{CT}$ management plans was assessed by biopsy when available, clinical follow-up, and survival analysis. Results: Stage was discordant on PET/CT and conventional imaging in $50.6 \%$ of patients ( $41.1 \%$ upstaged, $9.5 \%$ downstaged), with high management impact (change in treatment modality or curative intent) in $42.3 \%$ of patients. Both conventional imaging stage and PET/CT stage were strongly predictive of overall survival (OS) but there were greater differences between hazard rates and separations in the OS curves for stage groupings determined using PET/CT. OS was also strongly predicted by PET/CT-directed choice of therapy $(P<0.0001)$. Conclusion: $\mathrm{PET} / \mathrm{CT}$ frequently affects patient management and strongly predicts OS in NSCLC, supporting the appropriateness of such changes.

Key Words: non-small cell lung cancer; positron emission tomography; CT; PET/CT; diagnosis; treatment

J Nucl Med 2012; 53:1007-1015

DOI: 10.2967/jnumed.111.099713

\footnotetext{
Received Oct. 20, 2011; revision accepted Feb. 27, 2012.

For correspondence or reprints contact: Michael P. Mac Manus, Department of Radiation Oncology, Peter MacCallum Cancer Centre, St Andrew's PI., East Melbourne, Victoria, Australia 3002.

E-mail: michael.macmanus@petermac.org

Published online Jun. 7, 2012.

COPYRIGHT @ 2012 by the Society of Nuclear Medicine, Inc.
}

$\mathbf{P}$ ET has been rapidly adopted as a key imaging modality for the diagnosis and staging of lung cancer (1-4). Stand-alone PET scanners have been superseded by hybrid PET/CT machines that combine functional and structural imaging information, providing superbly detailed and accurate images (5). PET/CT is both diagnostically superior to older scanners (6) and faster. Despite its widespread use for cancer imaging in developed countries, there are ongoing questions regarding its cost-effectiveness $(7,8)$. These studies have generally focused on surgical patients for whom pathologic confirmation of diagnostic accuracy is available, but such patients actually reflect a minority of patients who could potentially benefit from more accurate staging (9). In patients receiving palliative radiotherapy or other noncurative treatments, detailed pathologic examination to confirm stage is seldom performed or considered clinically useful, and consequently the ability to validate the accuracy of staging techniques is compromised. Therefore, surrogates for diagnostic performance are required.

For individual patients, selection of ideal treatment and subsequent outcome in non-small cell lung cancer (NSCLC) depends critically on disease extent (10). More accurate characterization of stage should lead to avoidance of futile attempts at curative treatment and result in either improved prognostic stratification or no worse outcomes in individual stage groups despite less aggressive therapies. Accordingly, the purpose of this prospective clinical study was to evaluate both the clinical impact of incremental diagnostic information from PET/CT, compared with conventional imaging, and its prognostic significance based on overall survival (OS). Eligible patients in this study were consecutive patients with known or suspected NSCLC referred to our PET facility who were considered potential candidates for curative therapy with either surgery or radiation therapy (RT). Before the result was obtained, referring physicians indicated how the patient would be managed if PET/CT was unavailable, as described previously $(11,12)$. The study conditions reflect routine clinical use of ${ }^{18} \mathrm{~F}$ FDG PET/CT in a tertiary oncology facility wherein all 
clinical information at the time of PET reporting is integrated into the final conclusion regarding disease stage.

\section{MATERIALS AND METHODS}

\section{Patients}

From August 1, 2002, to July 31, 2003, all patients referred for PET/CT for evaluation of known NSCLC or suspected lung cancer and who were being considered for potentially curative therapies were invited to give written informed consent to participate in this ethics committee-approved study. When PET/CT was obtained before biopsy and the patient was subsequently found to have NSCLC, the PET/CT scan served the dual roles of diagnosis and staging. If biopsy after PET/CT showed no tumor, or malignancy other than NSCLC, the patient was not considered further in this analysis.

Referring clinicians completed a form defining the proposed management plan based on the pre-PET/CT diagnostic and staging information, including bone scintigraphy and MRI if performed. A CT scan of at least the chest and upper abdomen, performed within $60 \mathrm{~d}$ of PET/CT, was mandatory to exclude patients considered to have unequivocal evidence of disease unsuitable for treatment with curative intent. Bone scanning was done only to evaluate skeletal symptoms or if biochemical abnormalities suggested possible bone disease. After PET/CT, the referring clinician was contacted to discover the intended post-PET/CT management plan. Patients were followed up either until death or for a minimum of $5 \mathrm{y}$. Treatment actually delivered was recorded.

\section{PET/CT Protocol}

PET/CT images were acquired on a Discovery LS scanner (GE Healthcare). After $6 \mathrm{~h}$ or more of fasting, patients received approximately $370 \mathrm{MBq}$ of ${ }^{18} \mathrm{~F}$-FDG and rested for $1 \mathrm{~h}$ before PET and unenhanced CT from the skull base to mid thighs. Scans were interpreted with access to clinical information-previous investigation results including the diagnostic, contrast-enhanced CT of at least the chest and upper abdomen. As is usual in clinical practice, the PET/CT reader was not masked to other investigations because the purpose of this study was to assess the incremental rather than independent diagnostic value of ${ }^{18} \mathrm{~F}$-FDG PET/CT with the realization that there is already a selection bias intrinsic in the exclusion of patients with advanced disease on conventional imaging. Actual patient management was based on all available investigations including incremental information from PET/CT unless otherwise indicated. Patients at our facility are routinely discussed at a multidisciplinary meeting attended by medical, radiation, and surgical oncologists and cancer imaging specialists.

\section{Validation of PET/CT Results}

Although the purpose of this study was not to formally assess the comparative accuracy of imaging modalities, given the selection bias intrinsic in the referral of patients on the basis of conventional imaging findings, the validity of management decisions was determined by biopsy results, when available. When biopsy was unavailable, the following criteria were used in order of decreasing preference: additional imaging, therapeutic response evaluation, and clinical follow-up. Original diagnostic imaging reports were used to define conventional imaging and PET/CT staging, according to the TNM staging system of the American Joint Committee on Cancer (sixth edition) (13). The readers were thus agnostic to the primary endpoint of this trial, which was OS.

Individual lesions were considered positive on imaging if reported as positive for malignancy or deemed highly suggestive of malignancy. Because this study sought to evaluate the performance of ${ }^{18} \mathrm{~F}$-FDG PET/CT in routine clinical performance, there were no prescribed reporting criteria. However, PET/CT images were interpreted qualitatively, with sites of disease identified as ${ }^{18} \mathrm{~F}$-FDG-avid foci in a nonphysiologic distribution of intensity typically greater than adjacent normal tissues (and particularly higher than hepatic uptake) unless of small volume for which partial-volume effects needed to be taken into consideration. All reporting clinicians had at least $3 \mathrm{y}$ of experience in reporting ${ }^{18} \mathrm{~F}-\mathrm{FDG}$ PET and more than 6 mo of experience with reading PET/CT when the study commenced to minimize effects of a learning curve in what was a new modality at that time. Imaging studies were considered negative for malignancy if no abnormality was reported or if the lesion in question was reported as being consistent with a benign process. Imaging was considered equivocal if abnormalities could represent either malignant or inflammatory processes but biopsy was recommended for verification. Imaging results were assigned to true-positive, false-positive, or negative categories according to biopsy results or subsequent imaging, as outlined earlier in this article. These categories applied only to lesions not considered equivocal.

The following rules were applied to the imaging of distant metastasis: if imaging showed no metastatic disease at diagnosis and locoregional disease was controlled, metastasis within 6 mo was considered false-negative, and the absence of metastasis within 6 mo was defined as true-negative. For patients who did not obtain local disease control, the presence or absence of metastasis within the first 6 mo was considered nonassessable, because of the high risk of metastasis from their uncontrolled locoregional disease in this time interval.

A metastatic site was deemed true-positive if the biopsy result was positive, if an unequivocal therapeutic response was obtained at that site after treatment, or if subsequent disease progression occurred at the site. In equivocal cases, the lowest applicable stage was used, and uncertainty was recorded.

\section{Impact of PET/CT on Management}

The impact of PET/CT on patient management was defined using a previously published scale of impact (12) as follows: a change from one treatment modality to another (e.g., surgery to RT) or a change in treatment intent (curative to palliative) because of PET/CT represented high impact. A change in delivery of the same modality (e.g., a change in the RT target volume) represented medium impact. A low impact indicated no change in proposed management. If the PET/CT result was ignored (e.g., if resection was performed despite distant metastasis on PET/CT), impact was recorded as none.

\section{Treatment Policy}

Patients were managed according to the following broad treatment policies. Stage I-II NSCLC patients underwent surgical resections if medically fit and received definitive RT if they were not. Patients with stage IIIA and with stage IIIB who could safely be encompassed within a tolerable radiation target volume were offered definitive chemo-RT or definitive RT with curative intent, if they had a good performance status. Patients with disease too advanced for curative RT or with poor performance status were offered palliative treatments (palliative RT or chemotherapy) or supportive care. Patients rarely underwent mediastinoscopy before PET/CT because it is our policy to use PET results to select patients for mediastinal node sampling and to choose biopsy sites (14). 


\section{Statistical Methods}

All categoric data were summarized using counts and percentages. Statistical analyses were performed using 5\% 2-sided tests or the corresponding 2-sided 95\% confidence intervals (CIs). It was expected that more than $200 \mathrm{PET} / \mathrm{CT}$ scans for potentially curable NSCLC would be performed in the study period. In previous studies from our center, sample sizes more than 150 were sufficient to demonstrate robust survival differences between groups staged with PET $(15,16)$. OS was measured from the $\mathrm{PET} / \mathrm{CT}$ date to the date of death. Patients alive with more than $5 \mathrm{y}$ of follow-up were censored at $5 \mathrm{y}$. Kaplan-Meier curves of OS for stage and treatment groups were estimated and compared using the Mantel-Cox log-rank test, and relative hazard rates were estimated using Cox proportional hazards regression, with CIs derived by floating absolute risk methods.

\section{RESULTS}

During the study period, 296 patients with proven or possible NSCLC were enrolled. After patients ineligible for analysis were excluded, 168 patients with proven NSCLC remained. Reasons for exclusion were interval between $\mathrm{CT}$ and PET/CT more than $60 \mathrm{~d}(n=35 ; 27.3 \%)$ (17), final diagnosis not NSCLC $(n=81 ; 63.3 \%)$, overseas patients $(n=2 ; 1.6 \%)$, obvious distant metastasis on conventional imaging $(n=3 ; 2.3 \%)$, inadequate conventional imaging $(n=4 ; 3.1 \%)$, inadequate clinical information $(n=1$; $0.8 \%)$, or treatment that commenced before PET/CT $(n=$ $2 ; 1.6 \%)$. Of the 168 eligible patients, $115(68.5 \%)$ were men, and histologies were adenocarcinoma $(n=60 ; 35.7 \%)$, squamous carcinoma $(n=53 ; 31.5 \%)$, and large cell carcinoma $(n=19 ; 11.3 \%)$. The remainder were not further subclassifiable histologically $(n=31 ; 18.5 \%)$ or had rarer NSCLC subtypes $(n=5 ; 3.0 \%)$. Stage groupings by conventional imaging were stage I, $n=84(50.0 \%)$; stage II, $n=16$ (9.5\%); and stage III, $n=58$ (34.5\%). There were also 10 conventional imaging stage IV patients $(6.0 \%)$, either planned for aggressive management after resection of solitary brain metastasis $(18)(n=3)$ or with conventional imaging showing probable metastasis but with insufficient confidence to absolutely exclude potentially curative therapy $(n=7)$.

\section{Impact on Staging}

The median interval between CT and PET/CT was $25.5 \mathrm{~d}$. Comparison of PET/CT stage with conventional imaging stage, including substages, is shown in Table 1 . In only 83 cases $(49.4 \%)$ were conventional imaging and PET/CT stages concordant. Upstaging by PET/CT was more frequent than downstaging $(41.1 \%$ vs. $9.5 \%)$. Upstaging occurred in all pre-PET/CT stage groups but was most frequent in patients with conventional imaging stage II-III disease.

\section{Impact on Management}

Changes in patient management due to PET/CT and the overall impact of PET/CT are shown in Table 2. For 157 patients $(93.5 \%)$, referring physicians indicated that the pre-PET/CT management plan was either potentially curative treatment or an invasive biopsy to evaluate equivocal disease, usually in lymph nodes. In the remaining 11 patients $(6.5 \%)$, the proposed management plan was palliative, on the basis of equivocal conventional imaging, but curative therapy was being actively considered depending on $\mathrm{PET} / \mathrm{CT}$ results.

After PET/CT, the proposed management plan changed in 69 of 168 patients $(41.1 \%)$, and more than one third of patients in all conventional imaging stage groupings had high-impact PET/CT results (Table 3). The greatest effect was seen in conventional imaging of stage III $(51.7 \%$ high impact). Of 80 patients with a pre-PET/CT plan for curative surgery, 26 (32.5\%) had high-impact PET/CT results, usually because of a change in management to definitive RT $(n=9$, $11.3 \%$, including 1 patient also given definitive chemoradiation for a PET/CT-detected oropharyngeal carcinoma) or a change to palliative therapy $(n=13 ; 16.3 \%)$. The effect of PET/CT was greatest on candidates for radical RT. Of these 49 patients, 23 (46.9\%) had high-impact PET/CT results, usually because of upstaging. Of 48 patients actually given definitive RT, 7 (14.6\%) had significant alterations in target volumes because of PET/CT. Only 2 of 8 patients $(25.5 \%)$ being considered for palliative therapy had highimpact PET/CT results.

Twenty-two patients did not follow the post-PET/CT management plan stated by the referring physician after PET/CT, for reasons that included declining performance status $(n=10 ; 35.7 \%)$, refusing recommended therapy $(n=5 ; 17.9 \%)$, unresectability at thoracotomy $(n=2$; $7.1 \%$ ), and PET/CT findings being ignored by the treating physician $(n=5 ; 17.9 \%)$.

\section{Validity of Stage Assigned by PET/CT, Compared with Conventional Imaging}

Accuracy of PET/CT and conventional imaging staging was evaluated only for the small group of patients who were pathologically staged. Sixty-eight patients had surgery after PET/CT. Pathologic staging was assessable in 61 patients $(89.7 \%)$ and nonassessable in $7(10.3 \%)$ because of induction therapy after PET/CT or insufficient clinical information. In all 13 cases with available surgical staging for which the impact of PET/CT was high, PET/CT was correct. No tumor was found at PET/CT-negative sites with presumed metastasis on conventional imaging. Nodal disease was nonassessable because of inadequate sampling in 3 cases, and an unbiopsied distant suspected metastatic site was also nonevaluable. Despite PET/CT showing unresectable disease, surgery was performed in 2 cases. PET/CT findings were either accurate $(n=1)$ or nonassessable $(n=1)$. Six surgical patients $(9.8 \%)$ had false-positive nodes on PET/CT because of anthracosis $(n=3)$, sarcoidosis $(n=$ $1)$, reactive change $(n=1)$, or unknown cause $(n=1)$. Six $(9.8 \%)$ patients had false-negative nodes on PET/CT, and in 4 of these 6 the disease was microscopic only.

In the 107 patients without surgical pathologic staging, the validity of incremental PET/CT findings could be evaluated in only 33 (30.8\%), with PET/CT considered correct in $31(93.9 \%)$. 
TABLE 1

Comparison of Conventional Imaging and PET Stages

\begin{tabular}{|c|c|c|c|c|c|c|c|c|c|c|c|}
\hline \multirow{2}{*}{$\begin{array}{c}\text { Conventional imaging } \\
\text { stage group }\end{array}$} & \multicolumn{11}{|c|}{ PET stage group } \\
\hline & 0 & $1 \mathrm{~A}$ & $1 \mathrm{~B}$ & $2 \mathrm{~A}$ & $2 B$ & $3 \mathrm{~A}$ & $3 B$ & 4 & Total & Downstaged & Upstaged \\
\hline 0 & 0 & 0 & 0 & 0 & 0 & 0 & 0 & 0 & 0 & $0(0)$ & $0(0.0)$ \\
\hline $1 \mathrm{~A}$ & 2 & 24 & 3 & 1 & 0 & 3 & 1 & 9 & 43 & $2(4.7)$ & 17 (39.5) \\
\hline $1 \mathrm{~B}$ & 0 & 0 & 19 & 0 & 8 & 4 & 4 & 6 & 41 & $0(0.0)$ & $22(53.7)$ \\
\hline $2 \mathrm{~A}$ & 1 & 0 & 0 & 0 & 0 & 2 & 0 & 1 & 4 & $1(25.0)$ & $3(75.0)$ \\
\hline $2 B$ & 0 & 0 & 0 & 0 & 9 & 2 & 0 & 1 & 12 & $0(0.0)$ & $3(25.0)$ \\
\hline $3 A$ & 0 & 0 & 4 & 1 & 3 & 14 & 9 & 10 & 41 & 8 (19.5) & 19 (46.3) \\
\hline $3 B$ & 0 & 1 & 0 & 0 & 1 & 1 & 9 & 5 & 17 & $3(17.6)$ & 5 (29.4) \\
\hline 4 & 0 & 0 & 0 & 0 & 1 & 0 & 1 & 8 & 10 & $2(20.0)$ & $0(0.0)$ \\
\hline Total & & & & & & & & & & $16(10)$ & $69(41.1)$ \\
\hline
\end{tabular}

\section{PET/CT as Predictor of OS}

Median OS was 1.2 y (95\% CI, 1.1-1.5), and 60\% (95\% CI, 53\%-68\%) survived $1 \mathrm{y}$. During the median follow-up period of $5 \mathrm{y}, 127$ patients $(75.6 \%)$ died, giving a 5-y sur- vival of $24.4 \%$. All but 7 surviving patients had 5 y of potential follow-up. Both conventional imaging stage and PET/CT stage (Fig. 1) were strongly predictive of OS, but there were much greater differences between hazard rates

TABLE 2

Impact of PET/CT on Patient Management Plan

\begin{tabular}{|c|c|c|c|c|c|c|c|}
\hline \multicolumn{2}{|c|}{ Before PET/CT } & \multicolumn{2}{|l|}{ After PET/CT } & & & & \\
\hline \multirow{2}{*}{$\begin{array}{l}\text { Management } \\
\text { plan }\end{array}$} & \multirow{2}{*}{$\begin{array}{c}\text { No. of } \\
\text { patients }\end{array}$} & \multirow[b]{2}{*}{ Management plan } & \multirow{2}{*}{$\begin{array}{c}\text { No. of } \\
\text { patients }\end{array}$} & \multicolumn{4}{|c|}{ PET/CT impact } \\
\hline & & & & High & Medium & Low & None \\
\hline Observation & 1 & Surgery & 1 & 1 & 0 & 0 & 0 \\
\hline \multirow[t]{5}{*}{ Invasive biopsy } & 28 & Invasive biopsy & 9 & 19 & 0 & 9 & 0 \\
\hline & & Observation & 1 & & & & \\
\hline & & Surgical & 9 & & & & \\
\hline & & Radical $\mathrm{RT}^{\star}$ & 3 & & & & \\
\hline & & Palliative $^{\dagger}$ & 6 & & & & \\
\hline \multirow[t]{6}{*}{ Surgery } & 80 & Surgery & 54 & 26 & 0 & 50 & 4 \\
\hline & & $\begin{array}{l}\text { Diagnostic } \\
\quad \text { (invasive biopsy) }\end{array}$ & 2 & & & & \\
\hline & & Observation & 2 & & & & \\
\hline & & Radical RTł & 8 & & & & \\
\hline & & $\begin{array}{l}\text { Radical RT, for both NSCLC and } \\
\text { PET-detected pharynx cancer }\end{array}$ & 1 & & & & \\
\hline & & Palliative $\S$ & 13 & & & & \\
\hline \multirow[t]{6}{*}{ Radical RT } & 49 & Radical RT as planned\|" & 19 & 23 & 7 & 18 & 1 \\
\hline & & Radical CRT, field increased & 6 & & & & \\
\hline & & Radical CRT, field decreased & 1 & & & & \\
\hline & & Surgery & 4 & & & & \\
\hline & & Induction chemotherapy, followed by surgery & 1 & & & & \\
\hline & & Palliative chemotherapy or RT & 18 & & & & \\
\hline \multirow[t]{2}{*}{ Palliative } & 10 & Palliative chemotherapy or RT, as planned\# & 8 & 2 & 2 & 6 & 0 \\
\hline & & Palliative CRT, RT field increased & 2 & & & & \\
\hline Total & 168 & & 168 & $71(42 \%)$ & $9(5 \%)$ & $83(49 \%)$ & $5(3 \%)$ \\
\hline \multicolumn{8}{|c|}{$\begin{array}{l}{ }^{*} \text { Radical RT no chemotherapy }(n=1) \text {, radical CRT }(n=2) \text {. } \\
{ }^{\dagger} \text { Palliative chemotherapy and RT }(n=5) \text {, chemotherapy }(n=1) \text {. } \\
{ }^{\ddagger} \text { Radical RT alone }(n=1) \text {, radical CRT }(n=7) \text {. } \\
\text { §Palliative chemotherapy and RT }(n=10) \text {, chemotherapy only ( } n \\
\text { "Radical RT }(n=5) \text {, radical CRT }(n=14) \text {. } \\
\text { "Palliative chemotherapy and RT }(n=17) \text {. } \\
\text { "Palliative chemotherapy and RT }(n=5) \text {, chemotherapy only }(n=\end{array}$} \\
\hline
\end{tabular}


TABLE 3

Impact of PET/CT by Conventional Imaging Stage Group

\begin{tabular}{lcrrrrrrrrrr}
\hline $\begin{array}{c}\text { Conventional imaging } \\
\text { stage group }\end{array}$ & No. of cases & \% of cases & High & \% high & Low & \% low & Medium & \% medium & None & \% none \\
\hline 1 & 84 & 50.0 & 31 & 36.9 & 51 & 60.7 & 0 & 0.0 & 2 & 2.4 \\
2 & 16 & 9.5 & 6 & 37.5 & 10 & 62.5 & 0 & 0.0 & 0 & 0.0 \\
3 & 58 & 34.5 & 30 & 51.7 & 17 & 29.3 & 9 & 15.5 & 2 & 3.4 \\
4 & 10 & 6.0 & 4 & 40.0 & 5 & 50.0 & 0 & 0.0 & 1 & 10.0 \\
Total & 168 & 100 & 71 & 42.3 & 82 & 48.8 & 9 & 5.4 & 6 & 3.6 \\
\hline
\end{tabular}

and separations in the OS curves for stage groupings determined using PET/CT. For example, patients with conventional imaging stage IV had a 4.1 (95\% CI, 2.2-7.6) times greater odds of death than those with conventional imaging stage I. By contrast, patients with PET/CT stage IV disease had a $6.4(95 \%$ CI, 4.8-8.5) times greater odds of death than PET/CT stage I patients.

Median OS for 100 patients with conventional imaging stage I or II disease (anatomically resectable) before PET/ CT was 2.1 y (95\% CI, 1.5-4.0 y), with no difference in OS between stages I and II ( $P=0.86$, Fig. 2$)$. However, PET/ CT subdivided this same cohort of 100 patients into stages I-IV with markedly different prognoses between the PET/ CT stage groups. Patients with PET/CT stage I had a median OS of more than $5.0 \mathrm{y}$ (95\% CI, 3.0 to $>5.0 \mathrm{y})$, compared with those with PET/CT stage IV, with median OS of $0.7 \mathrm{y}$ (95\% CI, 0.6-1.2 y) $(P<0.0001)$.

For 131 patients who actually received the intended disease-modifying therapy after PET/CT, OS was strongly associated with the treatment modality (Fig. 3). Median OS was more than $5 \mathrm{y}$ after surgery $(n=61), 1.63 \mathrm{y}$ after definitive RT/chemo-RT $(n=25)$, and 0.88 y after palliative therapy (RT or chemo or both, $n=45)(P<0.0001)$. PET/CT stage grouping was also highly predictive of OS among patients who received either surgery or definitive RT. Both patients who underwent surgery despite PET/CT stage IV disease died within $1 \mathrm{y}$. The estimated OS of all PET/CT stage I patients at $1 \mathrm{y}$ was $97 \%$ (95\% CI, 91\%$100 \%$ ) and was superior to the OS of stage II and III patients, which was similar at $71 \%$ (95\% CI, 51\%-96\%) and $75 \%$ (95\% CI, 50\%-100\%), respectively.

\section{DISCUSSION}

Despite a rapidly growing body of literature (19-27) indicating that, compared with either conventional imaging or with stand-alone PET, PET/CT has superior diagnostic accuracy, especially for detecting distant nodal and systemic metastatic sites that are critical for selecting treatment and defining prognosis, the clinical utility of this new technology continues to be questioned by health funding agencies in some countries (8). Limitations of older single-modality ${ }^{18}$ F-FDG PET scanners included inadequate spatial resolution (28), poor localization of inflammatory uptake $(29,30)$, and restricted fields of view. The ability of PET/CT to fuse structural and functional information is a significant advance in cancer imaging. However, despite the fact that it was introduced into clinical practice around a decade ago, there has been a strong focus on proving that the technologic advances have translated into improved diagnostic accuracy, but remarkably little information exists on its capacity to appropriately change treatment decisions. In particular, relevant long-term follow-up data for patients with NSCLC are lacking even though existing publications provide a compelling rationale for the routine use of PET/CT staging.

In this study, we found frequent discordance between conventional imaging stage and PET/CT stage. Stage migration occurred in $50.6 \%$ of patients, primarily because of upstaging by PET/CT. Major management changes occurred as a result, both in the selection of treatment modality and in overall treatment intent. In $42.3 \%$ of our cohort, the impact of PET/CT was scored as high, indicating a change from one treatment modality to another, usually surgery to RT, or a change in intent from curative to palliative. Although upstaging was most frequent, 4 patients were appropriately offered surgery after downstaging by PET/CT. One patient received definitive chemoradiation for a PET/CT-detected oropharyngeal carcinoma. In patients treated with RT, the target volume was also often influenced by PET/CT. These results largely recapitulate our previous study using stand-alone ${ }^{18} \mathrm{~F}$-FDG PET.

In clinical practice, although biopsy is routinely obtained for diagnostic purposes, and suspected metastatic sites that would determine treatment strategy are also usually sampled, many patients are managed on the basis of conventional imaging that includes CT and other modalities as guided by the clinical scenario. Accordingly, many patients are treated on the basis of imaging studies that we know to be generally less accurate than ${ }^{18} \mathrm{~F}$-FDG PET/CT. However, in the absence of histopathologic confirmation of all potential sites of metastatic disease, how are we to assess whether management changes after PET/CT are appropriate? In the limited subset of those cases with available histologic confirmation, PET/CT was validated in more than $90 \%$, including all patients for whom PET/CT was discordant with conventional imaging. Of the 6 false-negative lymph node assessments made on PET/CT, 4 were due to microscopic disease and would have been undetectable by any other existing imaging technology. 


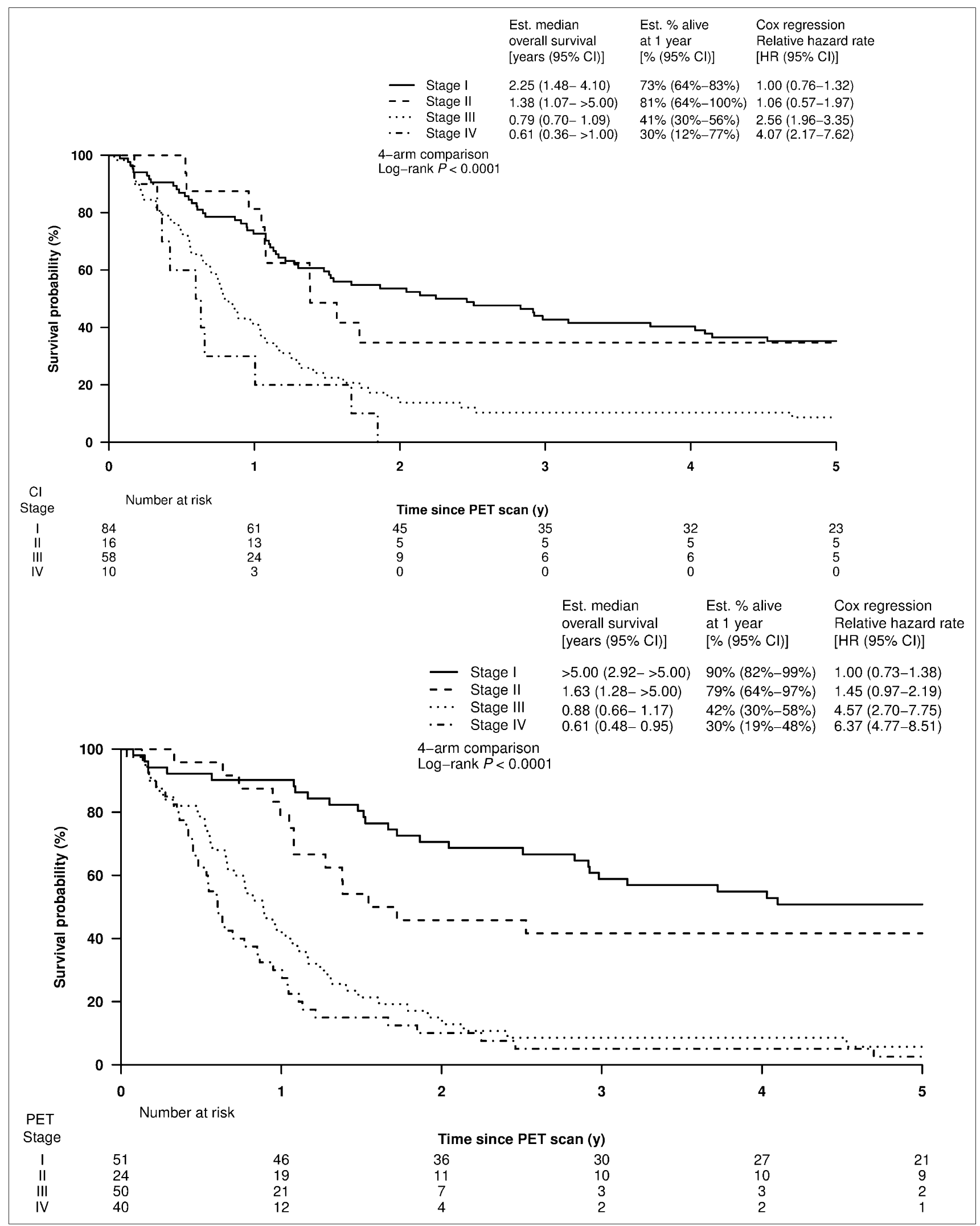

FIGURE 1. Survival according to stage groupings determined by conventional imaging (top) or PET/CT (bottom). Est = estimated. 


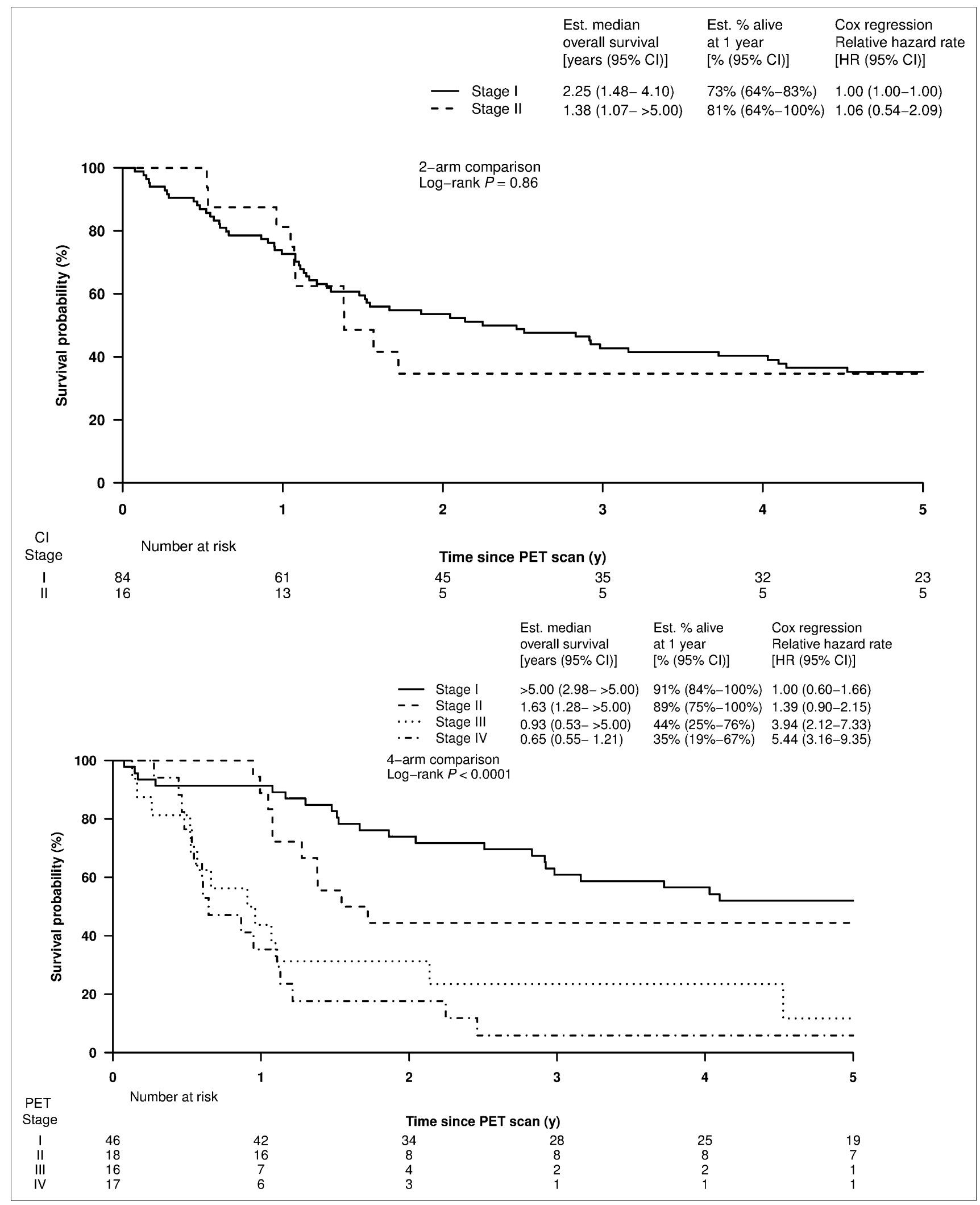

FIGURE 2. Survival curves for 100 patients found to have anatomically resectable disease by conventional imaging (stage I or II) according to their conventional imaging stage (top) or their PET/CT stage (bottom), showing prognostic stratification only by PET/CT stage. Est = estimated. 
FIGURE 3. OS by treatment actually received for 131 patients managed according to stated post-PET/CT plan. Est = estimated.

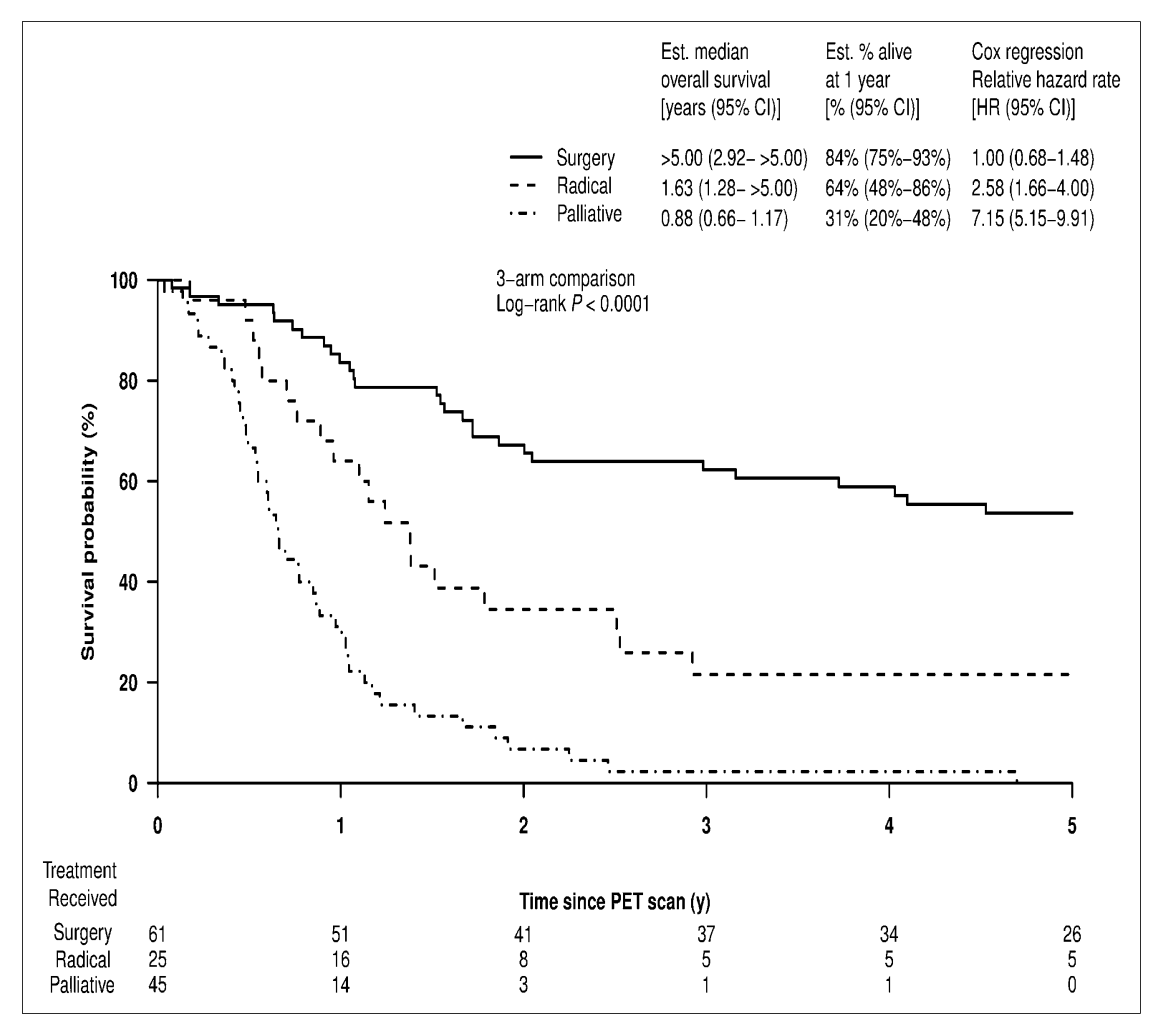

However, most patients with NSCLC are unsuitable for surgical resection and do not have comprehensive mediastinal staging. In nonsurgically staged patients, accuracy of clinical staging must usually be validated by follow-up imaging and subsequent clinical course $(25,26)$, although endoscopic ultrasound-guided biopsy can enable biopsy of previously inaccessible regions. When further imaging and follow-up were used to validate PET/CT and conventional imaging, PET/CT again proved superior and was estimated to be accurate in $94 \%$ of evaluable cases. Nevertheless, a criticism of such analysis will always be that there is a potential ascertainment bias, with only the sites most likely to contain disease being biopsied. Therefore, alternative surrogates of diagnostic accuracy are needed in cases in which comprehensive pathologic staging is impractical or impossible.

Another way of comparing PET/CT and conventional imaging is to consider the ability of each to predict survival. A more accurate test should provide better prognostic stratification, especially within a group of patients receiving the same type of therapy, thereby nullifying the prognostic implications associated with use of treatments with differential efficacy. In this study, PET/CT-based staging was significantly more powerfully correlated with OS than conventional imaging-based staging, across all stages and within cohorts of patients given any particular form of therapy. This finding is well illustrated by patients with conventional imaging stage I or II who were divided into stages I-IV by PET/CT, with wide differences in prognosis between the PET/CT stages but not the conventional imaging stages. This is a group of patients who would previously have been considered for surgical treatment. However, it must be conceded that the absolute differences in survival in the patients by stage based on conventional staging and ${ }^{18} \mathrm{~F}$-FDG PET/CT were small. These data suggest that the general ability of CT to stratify prognosis, which underpins the current staging groups, was achieved in this study and therefore the differences in stage cannot be explained by substandard conventional imaging. Moreover, ${ }^{18} \mathrm{~F}-\mathrm{FDG}$ PET/CT provided more robust separation of hazard ratios than conventional imaging in all situations evaluated. Further, despite applying less aggressive therapy to a significant number of patients, the survival across all stages in the PET/CT group was superior to those reported in the Surveillance, Epidemiology and End Results database for the same period.

Strengths of this study include prospective recording of the pre-PET/CT treatment plan and near complete followup for survival analyses. Major study endpoints (future management and OS) were unknown at the time of PET/CT reporting, limiting the possibility of bias. One limitation is the possibility of disease progression between conventional imaging investigations and PET/CT, although an interval of less than $60 \mathrm{~d}$ between CT and PET/CT scans was mandated, and the median interval was $25.5 \mathrm{~d}$. Although the lack of histopathologic staging information in all patients may be seen as a potential limitation, we believe that the fact that this study reflects routine clinical practice, for which a range of investigation may be used to reach sufficient diagnostic certainty to allow management planning, is actually an advantage in informing on the utility of this technique. As in clinical practice, conventional imaging 
and PET/CT images were not read with masking of other information because it is seldom that patients come to have PET/CT without first having had diagnostic CT. Patients with advanced disease will often not have PET unless this is done as a baseline for therapeutic response assessment. Similarly, patients with negative results on conventional imaging may also not be referred. Because of this selection bias, it is not appropriate to assess the diagnostic accuracy of PET/CT independently of CT. Although a randomization of patients to conventional imaging or ${ }^{18} \mathrm{~F}-\mathrm{FDG}$ PET/CT would address the question of whether the observed impact on management improves outcomes, we believe that denying patients access to PET in the staging setting would be unethical given the documented superiority of this technique in detecting occult sites of disease that have both management and prognostic implications.

Apart from the obvious patient benefits of appropriate choice of therapy, PET/CT could also have significant economic benefits. By preventing futile and morbid attempts at cure, PET/CT can spare scarce and expensive surgical and $\mathrm{RT}$ resources, allowing patients to receive more appropriate palliative therapies or supportive care. By reducing the time to definitive diagnosis and staging, PET/CT may improve the chances of cure by reducing the likelihood of progression before therapy (17). It is encouraging that the patients who had the most expensive and potentially morbid therapy or surgery had the longest OS, whereas those with the worst outcome had the least aggressive treatment.

\section{CONCLUSION}

PET/CT frequently and appropriately changes management in patients with NSCLC and predicts OS more accurately than conventional imaging. Economic modeling using prospective data such as these may allow valid estimates of the cost-effectiveness of PET/CT to be made (9).

\section{DISCLOSURE STATEMENT}

The costs of publication of this article were defrayed in part by the payment of page charges. Therefore, and solely to indicate this fact, this article is hereby marked "advertisement" in accordance with 18 USC section 1734.

\section{ACKNOWLEDGMENT}

No potential conflict of interest relevant to this article was reported.

\section{REFERENCES}

1. Hicks RJ, Kalff V, MacManus MP, et al. ${ }^{18}$ F-FDG PET provides high-impact and powerful prognostic stratification in staging newly diagnosed non-small cell lung cancer. J Nucl Med. 2001;42:1596-1604.

2. Kalff V, Hicks RJ, MacManus MP, et al. Clinical impact of ${ }^{18} \mathrm{~F}$ fluorodeoxyglucose positron emission tomography in patients with non-small-cell lung cancer: a prospective study. J Clin Oncol. 2001;19:111-118.

3. Vansteenkiste J, Fischer BM, Dooms C, Mortensen J. Positron-emission tomography in prognostic and therapeutic assessment of lung cancer: systematic review. Lancet Oncol. 2004;5:531-540.

4. Mac Manus M, Hicks RJ. The use of positron emission tomography (PET) in the staging/evaluation, treatment, and follow-up of patients with lung cancer: a critical review. Int J Radiat Oncol Biol Phys. 2008;72:12981306.

5. Freudenberg LS, Rosenbaum SJ, Beyer T, Bockisch A, Antoch G. PET Versus PET/CT dual-modality imaging in evaluation of lung cancer. Radiol Clin North Am. 2007;45:639-644.

6. Delbeke D, Schoder H, Martin WH, Wahl RL. Hybrid imaging (SPECT/CT and PET/CT): improving therapeutic decisions. Semin Nucl Med. 2009;39:308-340.

7. Schreyögg J, Weller J, Stargardt T, et al. Cost-effectiveness of hybrid PET/CT for staging of non-small cell lung cancer. J Nucl Med. 2010;51:1668-1675.

8. Buck AK, Herrmann K, Schreyogg J. PET/CT for staging lung cancer: costly or cost-saving? Eur J Nucl Med Mol Imaging. 2011;38:799-801.

9. Mac Manus MP, Hicks RJ. How can we tell if PET imaging for cancer is cost effective? Lancet Oncol. 2010;11:711-712.

10. Bordoni R. Consensus conference: multimodality management of early- and intermediate-stage non-small cell lung cancer. Oncologist. 2008;13:945-953.

11. Duong CP, Demitriou H, Weih L, et al. Significant clinical impact and prognostic stratification provided by FDG-PET in the staging of oesophageal cancer. Eur $J$ Nucl Med Mol Imaging. 2006;33:759-769.

12. Kalff V, Hicks RJ, Ware RE, Hogg A, Binns D, McKenzie AF. The clinical impact of ${ }^{18} \mathrm{~F}$-FDG PET in patients with suspected or confirmed recurrence of colorectal cancer: a prospective study. J Nucl Med. 2002;43:492-499.

13. Mountain CF. The international system for staging lung cancer. Semin Surg Oncol. 2000;18:106-115.

14. Hicks RJ, Lau E, Alam NZ, Chen RY. Imaging in the diagnosis and treatment of non-small cell lung cancer. Respirology. 2007;12:165-172.

15. Mac Manus MP, Hicks RJ, Ball DL, et al. F-18 fluorodeoxyglucose positron emission tomography staging in radical radiotherapy candidates with nonsmall cell lung carcinoma: powerful correlation with survival and high impact on treatment. Cancer. 2001;92:886-895.

16. MacManus MP, Hicks RJ, Matthews JP, et al. High rate of detection of unsuspected distant metastases by PET in apparent stage III non-small-cell lung cancer: implications for radical radiation therapy. Int J Radiat Oncol Biol Phys. 2001;50:287-293.

17. Everitt S, Herschtal A, Callahan J, et al. High rates of tumor growth and disease progression detected on serial pretreatment fluorodeoxyglucose-positron emission tomography/computed tomography scans in radical radiotherapy candidates with nonsmall cell lung cancer. Cancer. 2010;116:5030-5037.

18. Modi A, Vohra HA, Weeden DF. Does surgery for primary non-small cell lung cancer and cerebral metastasis have any impact on survival? Interact Cardiovasc Thorac Surg. 2009;8:467-473.

19. Lardinois D, Weder W, Hany TF, et al. Staging of non-small-cell lung cancer with integrated positron-emission tomography and computed tomography. N Engl J Med. 2003;348:2500-2507.

20. Pauls S, Buck AK, Hohl K, et al. Improved non-invasive T-Staging in non-small cell lung cancer by integrated 18F-FDG PET/CT. Nuklearmedizin. 2007;46:9-14; quiz N11-12.

21. Halpern BS, Schiepers C, Weber WA, et al. Presurgical staging of non-small cell lung cancer: positron emission tomography, integrated positron emission tomography/CT, and software image fusion. Chest. 2005;128:2289-2297.

22. Cerfolio RJ, Ojha B, Bryant AS, Raghuveer V, Mountz JM, Bartolucci AA. The accuracy of integrated PET-CT compared with dedicated PET alone for the staging of patients with nonsmall cell lung cancer. Ann Thorac Surg. 2004;78:1017-1023, discussion 1017-1023.

23. Shim SS, Lee KS, Kim BT, et al. Non-small cell lung cancer: prospective comparison of integrated FDG PET/CT and CT alone for preoperative staging. Radiology. 2005;236:1011-1019.

24. Hellwig D, Baum RP, Kirsch C. FDG-PET, PET/CT and conventional nuclear medicine procedures in the evaluation of lung cancer: a systematic review. $\mathrm{Nu}$ klearmedizin. 2009;48:59-69, quiz N58-59.

25. Billé A, Pelosi E, Skanjeti A, et al. Preoperative intrathoracic lymph node staging in patients with non-small-cell lung cancer: accuracy of integrated positron emission tomography and computed tomography. Eur J Cardiothorac Surg. 2009;36:440-445.

26. Carnochan FM, Walker WS. Positron emission tomography may underestimate the extent of thoracic disease in lung cancer patients. Eur J Cardiothorac Surg. 2009;35:781-784, discussion 784-785.

27. Krüger S, Buck AK, Mottaghy FM, et al. Detection of bone metastases in patients with lung cancer: ${ }^{99 \mathrm{~m}} \mathrm{Tc}-\mathrm{MDP}$ planar bone scintigraphy, ${ }^{18} \mathrm{~F}$-fluoride PET or ${ }^{18}$ F-FDG PET/CT. Eur J Nucl Med Mol Imaging. 2009;36:1807-1812.

28. Antoch G, Stattaus J, Nemat AT, et al. Non-small cell lung cancer: dual-modality PET/CT in preoperative staging. Radiology. 2003;229:526-533.

29. Shim SS, Lee KS, Kim BT, Choi JY, Chung MJ, Lee EJ. Focal parenchymal lung lesions showing a potential of false-positive and false-negative interpretations on integrated PET/CT. AJR. 2006;186:639-648.

30. Kim YK, Lee KS, Kim BT, et al. Mediastinal nodal staging of nonsmall cell lung cancer using integrated ${ }^{18} \mathrm{~F}$-FDG $\mathrm{PET} / \mathrm{CT}$ in a tuberculosis-endemic country: diagnostic efficacy in 674 patients. Cancer. 2007;109:1068-1077. 